\title{
Motor Education and Intervention Strategies for the Autistic Child, Modeling of the Psychomotor Therapist's Skills and Construction of the Intervention Plan.
}

\author{
Kacem Nejah ${ }^{1 *}$, Naffeti Chokri ${ }^{2}$, Grayri Ramzi $^{3}$, Elloumi $\mathrm{Ali}^{4}$
}

\author{
${ }^{1}$ Higher Institute of Sport and Physical Education of Sfax, Sfax, Tunisia \\ ${ }^{2}$ Higher Institute of Sport and Physical Education of Sfax, Sfax, Tunisia \\ ${ }^{3}$ Higher Institute of Sport and Physical Education of Sfax, Sfax, Tunisia \\ ${ }^{4}$ Faculty of Human and Social Science of Sfax, Sfax, Tunisia
}

Received Date: August 02, 2021; Accepted Date: August 18, 2021; Published Date: August 30, 2021

"Corresponding author: Kacem Nejah, Higher Institute of Sport and Physical Education of Sfax, Sfax, Tunisia. Email: kacemnejah@gmail.com

\section{Abstract}

The goal of our study is to empower people with autism to communicate and understand the world around them. It is about teaching skills and developing autonomy in life in society based on an education oriented to psychomotor intervention strategies while taking into account the particularities of the autistic person and the model of pedagogical and psychosocial skills of the teacher to create the best conditions, This by relying especially on the curriculum of the ESDM (Early start Denver model. Rogers, S., Dawson, G., 2013) [1] focused on the development of cognitive skills, including access to symbolism and communication for the construction of the intervention plan. Our work therefore ensures the development of a model of professional skills of the teacher in charge of building an intervention plan for autistic children in Tunisia. The aim of the programmed is to avoid this pathological process: The objective of this model is to reintegrate the child into the social loop, teaching him the basic elements of social life and filling the learning gaps that have accumulated over time The results show that the role of teachers tasked with developing the social and pedagogical skills of people with autism is based much more on the mode Active motor communication and adequate transfer of knowledge direct towards cooperation and understanding whose aim is integration and adaptation, learning is therefore a means and action of knowledge transfer which is only one component of the model of professional identification skills of the teacher.

Keywords: Autism; Education; Intervention plan; Intervention strategies; psychomotor; Skills modeling

\section{Introduction}

Formal education is based on the theoretical foundations of learning and the cognitive and behavioral approach, as well as on the knowledge and development of the child. Autism is a group of functional atypical whose educational strategies are in line with the child and the application of the child's personalized program ensures these principles during educational sessions. The educator is often integrated into the child care programmer. Integrated education for positive social interactions in young children presented by the Denver program and the ESDM (Early start Denver model) This Denver program: (Rogé, B., 2013) [1] was created in the eighties at the University of Colorado and is aimed at preschoolers, from two to six years old (Rogers, Herbison,
Lewis, Pantone and Reis, 1986; Rogers and Lewis 1989). This programmer integrates both educational and behavioral elements. It focuses on the development of cognitive skills, and on access to symbolism and communication. It is based on the practice of fun activities. Indeed, play is considered to be the support of the child's first learning, and in particular of social, emotional, communicative and cognitive skills (National research council, 2001). The child's education is thus integrated into social interactions, which are established between the child and the adult and which are described as positive. The development of socio-emotional skills involves experimenting with interpersonal relationships with adults, but also peer group sessions [2]. Play and positive interactions are the foundation of this program (Rogers, 2001). The role of the adult and the goals of the different play activities used depend on the objectives established in the child's personalized program, and vary according to his progress. ESDM: Early start Denver model [1].The ESDM is a program based on a developmental approach, where autism is considered a developmental disorder. This early program targets all developmental areas affected by autism. Hence the importance of analysis of interpersonal relationships, modulated by the quality of relationships and based on the implementation of positive effects, and on certain qualities of the worker such as receptivity, sensitivity, and reciprocity this model gives a great place to parents. The pedagogical practices used can be applied both individually and in groups in motor practice sessions. However, this requires training for all those concerned with the child. Moreover, it is a method that is part of interdisciplinary. The different professionals who follow the child will use these particular practices by sharing common goals. Within this multidisciplinary team, a person is designated as a resource person, who will work in connection with the family. This model was born from the discoveries made over the last twenty on the deficits present in autism at the level of: social orientation, attention sharing, and initiation of exchanges, imitation, communication, play and in the ability to learn. In addition, children with autism do not feel positive about social interactions. From 6- 9 months the child seems less in the interaction, causing a decrease in relational contact with his entourage. The child is increasingly withdrawn into isolation, says autistic. This has consequences on its neuronal and psychological development.

The exchange and development therapy (TED) presented for the first time by Lelord and Barthélémy in 1978, is based on a neuron functional and developmental approach to autism. It is part of an integrative care of the child with autism presented by various theoretical currents adapted to the child 
and his environment, the demands of the family and the resources of the different professionals. TED (exchange and development therapy) is defined as psychotherapy focused on the exchange and cognitive, emotional and emotional development of the child; it is based on a functional and developmental conception of autism, based on the neurophysiologic discoveries related to this pathology. Indeed, the deficits characteristic of autism are explained as the consequence of abnormalities in the functioning of neural networks responsible for social communication. Social communication is the ability to interact with an environment, in an appropriate way, in a specific social context. It includes several functions such as attention, imitation, intention, perception... The goal of EFILE is to re-educate these functions based on certain neurophysiologic principles: free acquisition, physiological curiosity and brain plasticity. EFILE is based on a functional analysis of the child's behaviors', based on his or her knowledge. Its objective is to promote the adjustments of the child, through a playful approach, to set up reciprocal and coordinated exchanges. The ESDM model of (Rogé, B., 2013) is conducted with the aim of evaluating the application of benefits to the child, it was carried out by Dawson et al in 2010 for two groups of children, of similar age one group having followed for two years the Denver Early Intervention Program (ESDM), and another having benefited from a conventional intervention, over an equal period of time. It is reported that children who completed the ESDM program showed a quantitatively more significant increase in intelligence quotient compared to children entering a conventional care program. The diagnosis of autism is in some cases modified (decrease in the degree of involvement). Another study was conducted in 2012 (Dawson et al, 2012) [2] showing the effect of the application of ESDM on neuronal mechanisms. The activity of children, who have benefited from the ESDM program for two years, is measured in front of a task of recognition of objects or face. The results then show that the cortical activation of these children is similar to that of children with typical development. Hence the application of ESDM to young children with autism, improves certain neural mechanisms responsible for adaptive behaviors. The application of ESDM has positive effects on the evolution of the developmental trajectory of the child with autism. These improvements in the various developmental areas, promoting a better quality of life. Several effective enforcement modalities are possible. For example, it has been shown that the application of this model was also effective in groups [3], if it is practiced intensively (between fifteen and twenty hours). Integrative management and exchange and development therapy within a global management of the child presented by (Blanc, et al, 2013) ensure an effectiveness of the model.

\section{Modeling the skills of the psychomotor therapist: Professional skills}

Competence is built from a field of activity, such as a trade. Each skill is unique since the skills of such a profession as the psychomotor therapist will not be similar to those of such another profession. For Martineau and Gauthier (2000), professional skills stem from knowledge of experience. For them, knowledge of experience is knowledge related to work tasks. It is mobilized on the ground and acquired in action. He is mobilized during interactions with students or other actors in education. Although competence may be the basis of an internal process, it is felt in external acts. It is observable and often, the skill seen in others seems simple because everything works without problems and without mystery. Its complexity implies a social recognition with regard to the competent person (Rey, 1998). Py and Leduc-Claire (2008) insist on the fact that professional skills are acquired through alternation and articulation of theory and practice. Professional practice would make it possible to highlight situations to be analyzed, which could be linked to theoretical notions. Thus, the construction of professional skills begins with practice after analyzing the situation concerned (Euzet, 1998). This during the practice is not only the terrain. It is also the place where the problems to be solved come from. The Boterf (1994) even speaks of competence in action since it considers that competences are always developed in practice. According to Perrenoud (1999), skills are composed of knowledge. Skills are capacities for action. The practitioner must be able to mobilize and update his knowledge and without this capacity, knowledge would remain mere knowledge. Rey (1998) shows the difference that remains between competence and knowledge; professional skills are a set of knowledge, knowhow, know-how that are linked to the practice of the teaching profession. Altet (1994) cited by Euzet (1998), professional competence is more precisely defined as the ability to mobilize these different resources mentioned above. Thus, professional competence can be considered as a high degree of adaptation to a given situation in the present (Euzet, 1998). Martineau and Gauthier (2000) argue that skills development builds professional identity. It is based on the development of professional skills that allow a mastery of the professional act as well as on the recognition of others. Indeed, this adaptation must be organized because in education, decisions must be made quickly (Gervais, Correa Molina and Lepage, 2008). According to Perrenoud (2006), it is the schemes that make it possible to organize resources and transform them into skills. Perrenoud (1994), quoted by Charlier (2001), considers that schemes use knowledge and transform it into skills. It is thanks to the schemes that knowledge is activated and allows skills to be updated. The Boterf (2000), resources can be personal (knowledge, know-how, skills, qualities, experiences, etc.) or environmental (material facilities, relational networks, etc.). The latter are divided into four categories, which are themselves divided into subcategories:

\section{Knowledge}

- Theoretical knowledge: Theoretical knowledge is divided into knowledge to be taught and knowledge to teach. The knowledge to be taught is the knowledge of the different disciplines to be transmitted to the students. The knowledge to teach, on the other hand, concerns the pedagogical knowledge that helps us for the interactive management of the classroom. This different knowledge's cannot be dissociated since they work together. Practical knowledge, on the other hand, comes from experience in the field and is also divided into two groups. Knowledge about practice is knowledge about the procedure to be adopted. Then, the knowledge of the practice is the knowledge coming from a successful practice (Bonnichon and Martina, 2008). Knowledge understands a situation. They can be concepts, disciplinary, organizational or relational knowledge.

- Environmental knowledge: knowledge related to context such as rules, organization, characteristics of objects.

- Procedural knowledge: knowledge that makes it possible to act according to methods aimed at achieving a goal.

- Know-how or operational capabilities o Formalized knowhow: approaches, instruments, methods mastered by the professional.

Empirical know-how: know-how drawn from lived experiences.

Cognitive know-how: intellectual operations allowing decision-making, analysis, invention or creation.

- Personal abilities or qualities: curiosity, strength, conviction, honesty, etc.

- Emotional resources: warning or encouraging signals that help guide one's action. Knowledge is therefore part of the development of professional skills (Altet, 2001).

The expected skills feiman-nemser and Buchmann (1987), cited by Boudreau (2001) believe that the purpose of teaching is to transmit knowledge and ensure that it is acquired. To do this, the teacher should have skills, such as an ability to interact and commit to the success of students' learning. Pou Boudreau (2001), the starting point for any teacher should be student learning. According to Clerc (1998), the work of a teacher is divided into three areas. 
- Pedagogical conduct: it gives rise to specific skills such as anticipation, organization, flexibility with regard to preparation; communication, decision-making and emergency management for the effective conduct of the class.

- The functions performed in the school: communicate, cooperate, negotiate with students, other teachers, parents, partners.

- Expertise: is in the order of the design, organization and conduct of learning situations. Some processes are conscious while others are unconscious and automatic.

- Social and relational skills: being able to cooperate, both in the classroom and in the school or even outside the school. These competences are divided into four branches which are as follows: being able to determine one's own position with regard to the social issues of the school; develop the attitudes of listening, facilitation and organization necessary both in the pedagogical relationship and in the work with the other adults of the establishment; use social know-how, negotiate, work in a team; assume professional deeds. Bélair (2001), too, divided the competences into five distinct fields:

- Class life: organization, schedule, space, choice of activities, etc.

- Relationship with pupils: communication, observation of difficulties, remedies, learning styles, differentiation, encouragement, individualization, etc.

- Subjects taught: appropriation of knowledge, content planning, knowledge of programmers, etc.

- Society: newsletters, meetings with parents, discussions with colleagues, innovation, etc.

- Person: know-how to be know-how to become, reflexive practice, decision-making, search for meaning, etc.

For Huber (2007), the skills of a teacher are as follows: diagnose, take into account representations, have positive expectations, use several pedagogical tools, associate students with the management of the course, evaluate, define objectives based on scholarly knowledge, know referential and programs, arrange his room, practice met cognition or reflexive analysis, promote autonomy, manage time, use teaching aids, create cognitive conflicts, create problem situations, create socio cognitive conflicts, engage, know how to complete your course, identify obstacles, leave your course unfinished, put your students in project, go from the complicated to the simple, go from simple to complicated, use group work, and others. To define professional skills, Perrenoud (2006), was inspired by the competency framework adopted in Geneva in 1996, to which he contributed greatly. To compile this list, he relied on the skills that are changing, the skills that have emerged in recent years and not basic skills such as planning or evaluation. The ten skill families according to Perrenoud (2006) are as follows. Each family includes several skills that are not presented here:

- Organize and animate learning situations

- Manage the progression of learning

- Design and evolve differentiation mechanisms

- Involve students in their learning and work

- Work in a team

- Participate in the management of the school

- Inform and involve parents

- Making use of new technologies

- Confronting ethical duties and dilemmas

- Manage your own continuing education

- The competency framework in force for Valais is divided into fifteen skills that are grouped into four fields of expertise. Each competency includes a number of indicators that allow bettering situating oneself in relation to the different fields

- Teaching and training
- Reflect on your practice

- Educate and train

- Work in teams

Obin (1998) proposes a model for classifying occupational skills into four types. The first two, the conceptual poles (theoretical knowledge) and functional poles (practical knowhow) are on the same axis, the culture of acquisition. A second axis, the culture of impregnation, also has two poles, the identity pole (integration and professional identity) and the personal pole (character and personality of each one). Often, these skills are involuntary and are present thanks to our own personal experiences The professional skills of the psychomotor therapist as a practitioner of the teaching profession is complex given the diversity of tasks; The simultaneity of tasks. actions and decisions in the present; uncertainty and the unexpected; the constant evolution of the articulation of teaching-learning, know-how and communication; adaptation; improvisation; rational thinking; the ability to mobilize resources; the use of routines; urgent decision-making; Class management, which is no longer an action in itself; a focus on student learning; the stabilization phase; the diversification phase; questioning; serenity and emotional distance; conservatism and complaints; and disengagement. Professional skills has a unique and specific approach to each profession; the skills that make up the professional identity are; self-confidence; the trust of others; control of the act; the recognition of others. Every psychomotor therapist wants to be a good teacher, but the characteristics vary depending on the nature of the activity and the nature of the learners, the qualities of what is called a good psychomotor therapist.

Friendliness be friendly and friendly with his students. It is a plus if his students can share their problems with him, without fear or hesitation. A good personality Students are always attracted to teachers with a good personality, which improves communication, understanding and, ultimately, good results. Deep knowledge and good education another important feature of a good psychomotor therapist is his knowledge and his own education. A good listener a good psychomotor therapist should have the characteristic of being an even better listener. A good sense of humor a good psychomotor therapist should also have the quality and characteristic of having a good sense of humor. Kindness one of the most important characteristics to have.

\section{Methodology}

\section{The Denver Program}

The Denver program was created in the eighties at the University of Colorado. It is aimed at preschool children, from two to six years of age. This programmer integrates both educational and behavioral elements. It focuses on the development of cognitive skills, including access to symbolism and communication. This model is based on the practice of fun activities. Indeed, play is considered to be the support of the child's first learning, and in particular of social, emotional, communicative and cognitive skills (National research council, 2001). The child's education is thus integrated into social interactions, which are established between the child and the adult and which are described as positive. The development of socio-emotional skills requires bias in experimenting with interpersonal relationships with adults, but also with peers (group sessions). Play and positive interactions are the foundation of this program (Rogers, 2001). The role of the adult and the goals of the different play activities used will depend on the objectives established in the child's personalized program, and will vary according to his progress. ESDM: early start Denver model [4].

\section{Protocol Experimental}

The ESDM methodology is an evaluation tool whose criteria are based on the sequences of skills development belonging to eight different domains: language pouring on the 
receptive and expressive, social interactions, global motor skills and fine motor skills, imitation, cognition, and play skills. This curriculum consists of 480 items organized into 4 levels: several periods from 9-12 months to 48 months (first level 9/12-18 months, second level 18-24 months, third 24-36 months and fourth level 36-48 months). This tool has been designed specifically for children with ASD. The organization of the items and their place in the different levels was determined by research on the typical development of the child, and by clinical observation of the development of the child with ASD. The curriculum is administered to the child either by a single professional or by the entire interdisciplinary team that shares the different fields, and this every twelve weeks. The administration must be similar to the intervention: that is to say, it is necessary to structure the room as for the care, and to set up an interactive style based on the game (choice of materials and favorite activities). All areas should be assessed; items at the lower or higher level should be examined if necessary. Parents are asked to provide information: on the child's skills, on the routines he knows his favorite activities by direct interview. The behaviors thus observed are coded according to a defined code, then quoted and finally compared to a checklist. Mastery of learning practices: The ESDM uses the principles of different methods. Indeed this model borrows certain principles belonging to the DENVER method [3]: the modulation of the awakening of the child, the dyadic commitment, the quality of the elaboration of routines, the combination of the different objectives, the use of communication in all activities, varying the functions, promoting non-verbal communication, and the use of the one more word rule (Use one more word than the number of words the child uses). It also uses TRP principles: follow the child's example, give him or her choice (activities, materials), use the tour de rôle, and reinforce all the child's attempts. Finally, he also uses the principles of the $\mathrm{ABA}$ : attention, the $\mathrm{ABC}$ format (SRC in French).The application of behavioral teaching techniques, the repetition of actions, and the management of undesirable behaviors.

\section{Teaching Framework and Use of Joint Activities}

The child and the therapist carry out together, from session to session, the same routine activities. These routines are shortterm activities ( 2 to 4 minutes) that are selected according to the preferences, and interests of the child. They have several objectives, in different areas. The implementation of routines goes through several stages: from the choice of activity, to the learning of the routine, by developing the turn of role and collaboration between the two protagonists, to the complexity of the routine action to expand the repertoire of activities of the child and develop his mental flexibility, until the cessation of the routine, to move to a new routine. There are several types of routines. Routines with objects make it possible to work on the alternation of attention, on the triadic commitment (child-object-therapist) and joint attention. Sensory routines are part of a positive effect, in order to promote dyadic engagement, in particular through the sharing of pleasure activities. These sensory routines make it possible to modulate the child's awakening and attract his attention, to promote reciprocity and social exchanges. The objectives pursued through these different routines are the development of communication, cognitive and motor skills, but also to promote imitation, joint attention and exchange. To summarize the pedagogical practices used in the ESDM [5]. Are based on the management of the child's attention, the modulation of his affect and awakening, the optimization of his motivation, but also on the quality of the dyadic commitment, in a positive effect. The adult must be sensitive and receptive to the child, and adapt to the child's language skills. The activities chosen must promote joint attention and cognitive development. Learning is based on the implementation of routines and repetitions, but also by the introduction of variations within these routines. Nevertheless, it happens that despite the respect of all these principles the child does not progress. In this case, this model established a decision tree, a hierarchy of measures to be implemented according to the situations.

\section{Mode of Investigation}

The sessions are carried out in a shared game environment without any learning constraints. They are predictable with the introduction of routines, safe reference points for the child. The sessions are often biweekly and of short duration of thirty minutes each, during which the activities are supervised by two people, who alternate between the role of observer and that of psychomotor therapist. The intervention was carried out through the functional assessment of the child and the creation of an individualized project. The functional evaluation of the child guided us to structured the detailed developmental phases of the child by detecting the strengths, and still fragile points, the emerging skills to be consolidated. The assessment focuses on the examination of: autistic symptomatology (ECA-R), disorders of psycho physiological functions, cognitive functioning, language or socio-communicative skills Vismara, L., Young, G., \& Rogers, S. (2012) [6]. This assessment also makes it possible to know what the child appreciates in order to define the activities that will be proposed to him during the sessions. The educational provisions drawn up are regularly reviewed following reassessments of the child. It is about conceptuality the environment so that the child is in the best possible conditions promoting his motivation to learn, and developing his skills. The principles are necessary: serenity, the availability of the psychomotor therapist, and reciprocity. Serenity is based on the establishment of a secure atmosphere. The objectives and effects of EFILE on the development and behavior of children with autism is combined with a comprehensive care programmers focused on the child, his or her educational environment and the nature of his or her interaction with the psychomotor therapist. The goals of the combination of these interventions are to homogenize the psychological development of the child, the installation of emerging skills and pivotal functions to improve the socio-emotional adaptation of the child in his environment, and improve his integration into society.

\section{Study Population}

The study concerns 25 children between 4 and a half and 6 years old, cared for at the private center of the autistic child in Sousse, benefiting from TED session several times a day. This over a period of 3 months. An initial assessment is conducted and then a reassessment takes place at the end of this period. This re-evaluation is to check if an improvement in capacities: imitation, shared attention, interaction, regulation of behavior, perception has been made. In addition, regular evaluation of autistic symptoms to show if a decrease in social communication disorders is achieved. The analysis of the child's interaction with the environment will be observed considerably to check whether the children accept the novelties of the environment, and better match their behavior. These testify to the improvements following this intervention, promotes the development of the child. Perrault (2010) builds his own tool to support the tasks and situations that teachers face. This tool runs on 10 skills. These competencies are categorized into 3 types of competencies that are respectively competencies of transversal competencies, at the heart of the profession and academic skills. Among the competencies "at the heart of the job" is the seventh competency "evaluating students". According to Gérard's methodology (2003) to assess the effectiveness of a training, the respondent do selfassessment of mastering these competencies which consists in estimating his level of effectiveness on a scale of 1 to 8 boxes ranging from "not at all effective" to "Quite effective ' for each of the academic competencies, at the core of the business and transversal before and after the Training. Using the 10 skills, he built a training evaluation scale. The scale consists of 33 statements where the teacher is asked to indicate his opinion on a Likert scale ranging from 1 (quite disagree) to 8 (fully agree) (see appendix). According to Gerard (2003), each participant "agrees to take up the different objectives of the training, formulated from the skills referred to in vocational training, and to ask the participant to estimate for each 
objective his level of Competence before and at the end of the Training. The validation we made of the tool showed us that this double questioning can be done at the same time, at the end of the Training. This questionnaire is composed of 10 dimensions, represents the 10 competencies, and each competency has statements according to the (Table 1) below. The skill to be evaluated in this research is the competency "evaluating students", which is a competency in the core of the profession that touches the act of Teaching.

\section{Results}

Our results show that at the functional level, there are deficits in social communication channels. Indeed the disorders of social communication present in people with autism are due to abnormalities of the functions of decryption of the data transmitted by others. To promote communication, learning and the homogenization of the child's development. The processing of information, and the developmental shifts associated with the objectives of these interventions seem to be in line with the role of the psychomotor therapist. In addition, these interventions are based on certain processes that are defined as the ability of neural networks to modulate according to the experiences that the child makes. It seems more important. These interventions are based on certain processes of skills and management of intergroup and intragroup relationship (Table 1) presents the variation of functions determining the nature of communications of autistic subjects with each other and with the psychometrical, so the functions are expressed by:

- The semantic function whose non-verbal communication transmits a certain amount of information is independent of the verbal (symbolic gestures, iconic and clues).

The syntactic function or the non-verbal intervenes at the level of the segmentation of the spoken string, and at the level of the synchronization of verbal and non-verbal signs.

The pragmatic function that distinguishes three functions influencing the course of a conversation

The expressive function, which provides information on The interlocutor (voice, clothes, gaze..

- The appetite function, which reflects the motivations, goals or intentions of speaker behavior.

The reactive function, which concerns all short and fast reactions of the receiver, informing the transmitter about the effectiveness of its communication.

\section{The variation of determining functions the nature of communications of autistic subjects with each other and with others}

\begin{tabular}{|c|c|c|c|c|c|c|}
\hline & & sum & ddl & Medium & $\mathrm{F}$ & Sig. \\
\hline \multirow{3}{*}{$\begin{array}{l}\text { The semantic } \\
\text { function }\end{array}$} & Intergroup & 10,153 & 23 & 0,441 & 3,531 & 0,4 \\
\hline & Intragroups & 0,125 & 1 & 0,125 & & \\
\hline & Total & 10,278 & 24 & & & \\
\hline \multirow{3}{*}{$\begin{array}{l}\text { The syntactic } \\
\text { function }\end{array}$} & Intergroup & 4,684 & 22 & 0,213 & . & . \\
\hline & Intragroups & 0 & 1 & 0 & & \\
\hline & Total & 4,684 & 23 & & & \\
\hline \multirow{3}{*}{$\begin{array}{l}\text { The pragmatic } \\
\text { function }\end{array}$} & Intergroup & 9,097 & 23 & 0,396 & 1,139 & 0,641 \\
\hline & Intragroups & 0,347 & 1 & 0,347 & & \\
\hline & Total & 9,444 & 24 & & & \\
\hline \multirow{3}{*}{$\begin{array}{l}\text { The expressive } \\
\text { function }\end{array}$} & Intergroup & 30,704 & 23 & 1,335 & 96,117 & 0,08 \\
\hline & Intragroups & 0,014 & 1 & 0,014 & & \\
\hline & Total & 30,718 & 24 & & & \\
\hline \multirow{3}{*}{$\begin{array}{l}\text { The appetite } \\
\text { function }\end{array}$} & Intergroup & 4,971 & 23 & 0,216 & 6,917 & 0,293 \\
\hline & Intragroups & 0,031 & 1 & 0,031 & & \\
\hline & Total & 5,002 & 24 & & & \\
\hline \multirow{3}{*}{$\begin{array}{l}\text { The reactive } \\
\text { function }\end{array}$} & Intergroup & 5,93 & 23 & 0,258 & 3,667 & 0,393 \\
\hline & Intragroups & 0,07 & 1 & 0,07 & & \\
\hline & Total & 6 & 24 & & & \\
\hline
\end{tabular}

Table 1: The variation of determining functions the nature of communications of autistic subjects with each other and with others.

The results of the study showed that $72 \%$ of EDSM participants have higher activation when viewing objects. ESDM children have also demonstrated a faster response to social stimuli. In addition, this study found a correlation between higher levels of activation for social stimuli and higher levels of social engagement. However, although there were significant differences between the ESDM and control groups in terms of social activity, there was no correlation between these outcomes of language and adaptive behavior. Three children in the ESDM group no longer met the criteria for a diagnosis of autism, however these changes were considered not significant. There was no significant difference. Adaptations of Denver early start model in which parents were trained to implement the intervention. In our study, parents received 20 to 30 minutes per week of training, coaching and feedback on their implementation of ESDM teaching techniques over 6-week periods. The parents then used the skills they learned with their own children at home. However, it should be noted that parenting skills improved compared to référence.la (Figure 1) show this variation determined by communications functions

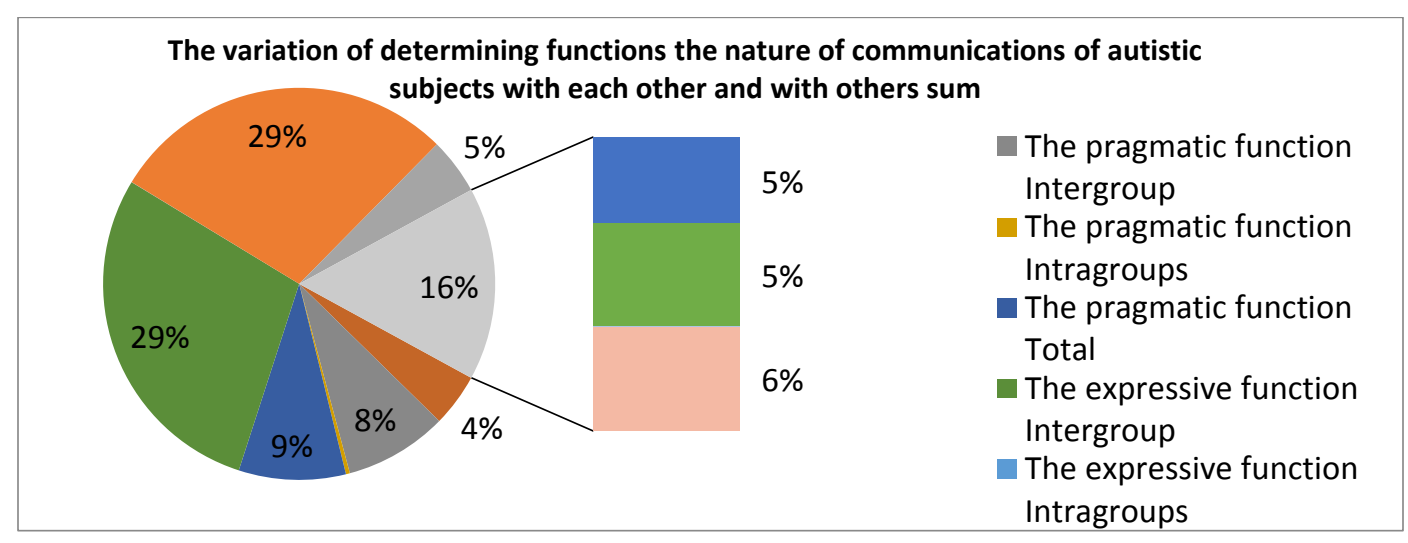

Figure 1: The variation of functions determining the nature of communications of autistic subjects among themselves and with others. 
The analysis of the educational functions of the psychomotor therapist shows that the difficulties of communication and social interactions express a feeling of loneliness The classic behaviors allowing friendship and sharing, reciprocity or cooperation are not natural. Autistic children play in an idiosyncratic way, that is to say that they have their own way of playing a game, this one is often unconventional and slows down the entry into a relationship with the other. Autistic people have difficulty identifying the roles of transceivers in a conversation with others. They also make few comments on what their interlocutor says. They do not give information about their level of understanding or misunderstanding of a message and little support the words of others with reminders or confirmatory sentences. Communication and social interactions then give the impression of being a one-way street and the autistic person seems to be a passive receiver. This during the relevant information is among several proposed in a conversation. They may repeat the same information several times. Autistic people have difficulty adapting the communication style and/or language level. The beginning of the language is difficult: starting a conversation, knowing how to initiate the interaction requires an effort of reflection and is not natural. Group conversations are more difficult to understand than individual conversations. In such a situation the autistic person seems to intervene impromptu, on subjects unrelated to the main conversation or on subjects out of step with the conversation. Understanding and using implicit notions or innuendos is also complex for autistic people and makes communication and social interactions difficult. If communication and social interactions differ in their use among autistic people, these differences do not always have a negative value, and it would be simplistic to perceive this communication only as a symptom or dysfunction that needs to be corrected. To determine a model of professional skills of the psychomotor therapist and autistic children a methodology and working tools enriching psychomotor practice such as the evaluation tools of these interventions that can be used to complement the observation of the psychomotor therapist (ESDM curriculum, scale of autistic behaviors used in TED). These tools make it possible to have a complete profile of the child in the different developmental areas. In addition, they also make it possible to give the psychomotor therapist a certain methodological rigor in his work. Thus the psychomotor therapist seems to have a legitimate place in the project of care of the child with autism. Nevertheless, the psychomotor intervention with the autistic young child requires an important commitment of the psychomotor therapist and his body which stimulates a balance of his professional skills identify by a model based on friendliness, good personality, deep knowledge of the specificity of education, the good listener, the bearer of a good sense of humor and kindness. It must also be a good listening to the proposals of the child, to strengthen and support him. It must also maintain a positive relational climate. The psychomotor therapist is a key player in the care of children with autism. It aims at the development of the child in areas specific to psycho motricity such as sensor motor skills, exploration behaviors, tonic-emotional adjustments and cognitive skills. The interventions aim to promote sensorimotor and emotional processes of exchange and development based on knowledge of the alteration of certain brain mechanisms causing deficits in the development of intentional motor sensor city. The training of neurophysiologic functions is therefore conducive to the installation of prerequisites, exchange and communication, thanks in particular to cerebral malleability. The game, the shared pleasure, is the mediators of the training of these altered functions. These are tools that the psychomotor therapist often uses intuitively. These interventions focus on the establishment of a positive relationship between the child and the therapist, to promote the momentum towards communication. That is to say, to offer the child a maximum of sensor motor, pleasant and motivating experiences, in a positive social context, in order to bring the child into communication and exchange with others; This during the young person with autism benefits from a rehabilitation of social psycho motricity (imitation, motor synchronization, non-verbal communication, associated with work on the regulation and sharing of emotions. These interventions therefore seem to provide a theoretical basis for the intuitive practice of the psychomotor therapist.

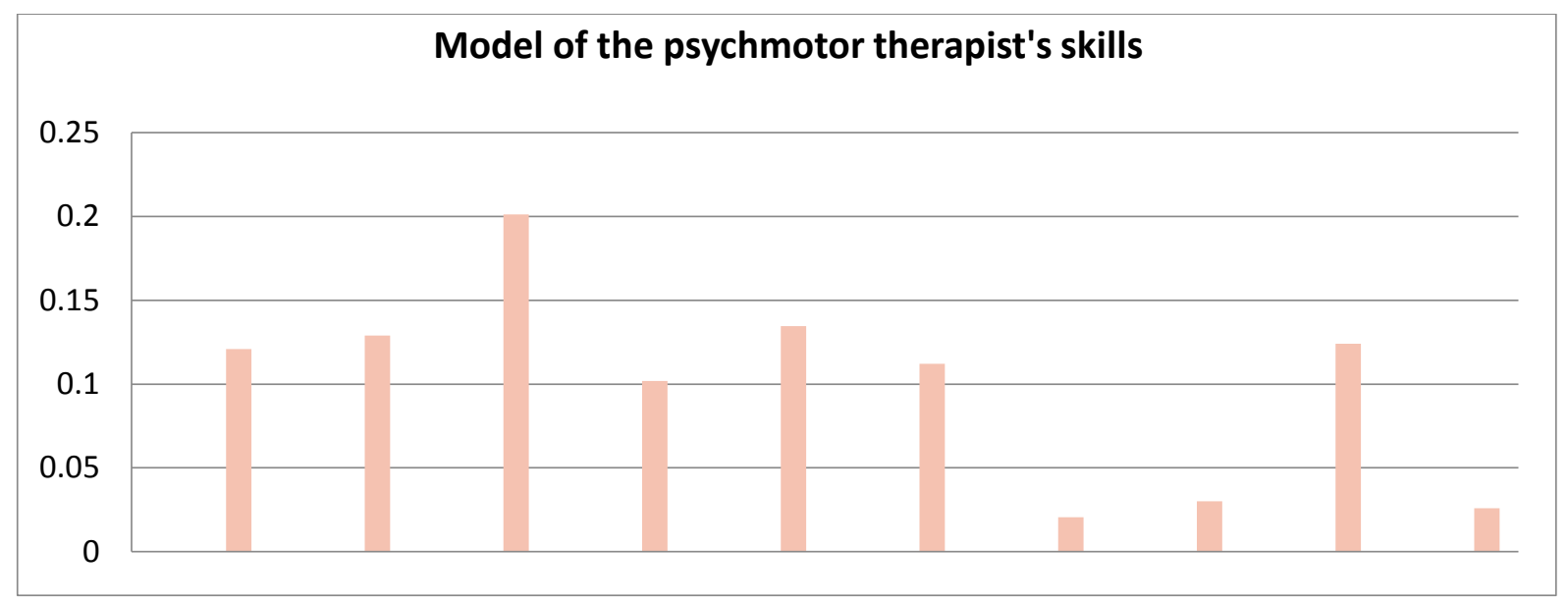

Figure 2: Intervention strategies and model of the psychomotor therapist's skills.

According to (Figure 2), we see that there are 4 skills that determine the model of professional skills of the psychomotor therapist intended to teach autistic children, they are respectively skills $1,2,3$ and 5 :

$\checkmark \quad$ Skill 1: act as a public servant and in an ethical and responsible manner $(12.10 \%)$

$\checkmark \quad$ Skill 2: Mastering the language to teach and communicate $(12.9 \%)$

$\checkmark \quad$ Skill 3: master the disciplines and have a good general culture $(20.12 \%)$

$\checkmark \quad$ Skill 5: organize class work (13.45\%)

The values recorded for the 6 skills fulfilling the professional skills model of the educator of autistic children are respectively: $\checkmark \quad$ Skill 4: Design and implement your teaching (10.18\%)

$\checkmark$ Skill 6: Take into account the diversity of students (11.20\%)

$\checkmark \quad$ Skill 7: evaluate students $(2.05 \%)$

$\checkmark$ Skill 8: Mastering information and communication technologies $(3.01 \%)$

$\checkmark \quad$ Skill 9: work as a team and cooperate with parents and school partners $(2.4 \%)$

$\checkmark \quad$ Skill 10: training and innovation (2.59\%)

Hence the results of the interview with the educator in charge of psychomotor work tasks prove an orientation towards skills of responsibility, communication of mastery of the activity and organizations 


\begin{tabular}{|l|l|l|}
\hline & $\begin{array}{l}\text { Standard } \\
\text { deviation }\end{array}$ & $\%$ \\
\hline act as a public servant and in an ethical and responsible manner & 1,45 & $12,10 \%$ \\
\hline Mastering the language to teach and communicate & 1,45 & 0,072 \\
\hline master the disciplines and have a good general culture & 1,37 & $12,90 \%$ \\
\hline Design and implement your teaching & 1,31 & $20,12 \%$ \\
\hline organize class work & 1,36 & 0,073 \\
\hline Take into account the diversity of students & 1,52 & $13,18 \%$ \\
\hline evaluate students & 1,33 & 0,068 \\
\hline Mastering information and communication technologies & 1,52 & 0,068 \\
\hline work as a team and cooperate with parents and school partners & 2,03 & 0,079 \\
\hline training and innovation & 1,65 & $3,01 \%$ \\
\hline
\end{tabular}

Table 2: The skills of the psychomotor therapist.

From the table of the interview on competence 4 (design and implement one's teaching), (Table 2) presents the ideas that show the frequency of use of the lexical fields of competence 4 during the interview. From the results of the interview for the psychomotor therapist, it is noted that there is a homogeneity of response of $(10.18 \%)$ with regard to a lack of mastery of the fourth skill.. From the above interview table we have identified the lexical fields that agree with competence 6: "take into account the diversity of students" are essentially (diversity, heterogeneity, test, measures, work by workshop, groups of levels, pedagogical groups, differentiated group, various performance, sexual diversity, social diversity, psychological diversity, mental diversity, cultural diversity ...). Based on the results of the interview, there is homogeneity of response of $(11.20 \%$ ) with regard to a lack of mastery of the sixth competence "take into account the diversity of students". Hence the mastery of this skill is dependent on the mastery of its components. For competence 7 "evaluate students" are essentially (evaluation, observation grid, objective evaluation, subjective evaluation, predictive evaluation, summative evaluation, precise evaluation, clear evaluation, test beginning of cycle, test end of cycle,). The results prove ideas that show the frequency of use of 7 skill lexical fields during maintenance. From the results of the interview it is noted that there is heterogeneity of response of $(2.05 \%)$ hence a lack of mastery of the seventh competence "assess students". So the psychomotor therapist does not master this skill .the lexical fields that agree with the skill 8: master the technologies of information and communication (ICT, computer, email, social networks, music, photos, videos, cameras...). Based on the results of the interview, it is noted that there is heterogeneity of response of $(3.01 \%)$ with regard to mastering the eighth skill "mastering information and communication technologies". So the use of this skill is negligible for the children's teacher autistes.la skill 9: working in a team and cooperating with parents and partners of the school are (working in a team, partner of the school, parent, the supervisor, the student, the trainee, the father, the mother, the counselor, the institution, the advisor,...). We note that there is a heterogeneity of response of $((2.4 \%)$ with regard to a lack of mastery of the ninth skill "work in a team and cooperate with parents and partners of the school". So the psychomotor therapist has not mastered this skill despite the importance given to the application of the ESDM: early start Denver model. Finally the skill 10 "to train and innovate" which are essentially (to train, to innovate, motivation, seminar, guide for trainee, luggage, creativity,). Based on the results of this, there is heterogeneity of response of $(2.59 \%)$ hence a lack of mastery of the tenth skill "to train and innovate".

\section{Discussion}

The objective of our study was to provide the autistic person with the means to communicate as well as understanding and adaptation with the world around him. The determination of the model of professional skills of the teacher and development of autonomy to life in society base on an education oriented to psychomotor intervention strategies while taking into account the particularities of the autistic person and the model of pedagogical and psychosocial skills of the teacher to create the best conditions, This relying especially on the curriculum of the ESDM (Early start Denver model. Rogers, S., Dawson, G., 2013) [4] focused on the development of cognitive skills, including access to symbolism and communication for the construction of the intervention plan [7]. Our work has therefore ensured the development of a model of professional skills of the teacher in charge of building an intervention plan for autistic children in Tunisia. The results showed that the role of teachers entrusted with tasks of developing the social and pedagogical skills of people with autism is based much more on the active mode of motor communication and adequate transfer of knowledge oriented towards cooperation and understanding whose goal is integration and adaptation, learning is therefore a means and action of knowledge transfer which is only one component of the model of identity skills vocational training of the teacher. The objectives of our research are based on the child's skills and abilities. The functional objectives were oriented towards actions of adaptation and functional analysis of the expected behavior to ensure following the identification of the action of the teacher with a view to highlighting the teaching practice hence the identification of the behavior in its generalization, its initiation, its spontaneity. And then determine the criterion for this behavior the use of the Denver early start model agrees that it is necessary to have more data with better methodological rigor in order to create a stronger evidence base to support the intervention [8]; Warren et al., 2011; Waddington et al. 2016). The study is therefore an important contribution to the field of early intervention research on autism and has many design strengths, including the appropriate intervention plan and structured education. However, our results are consistent with Combine's research, which also identifies several factors that could threaten the internal and external validity of the study results. She points out that while some of the gains made by the ESDM group were statistically significant; the magnitude of these gains was moderate. The use of adaptive score theory due to increased age of participant's remains necessary, to ensure that there has been no real increase in the rate of development. Most of the gains are attributable to language, from where the use of the study of the mastery of skills of the educator and the good management of the know-how and be, it is noted that the measurement tools used did not involve, for the most part, the direct observation of the participants, but rather relied on the report of the parents present by the competence 9 which is not well mastered on the part of the educator that can influence the responses. The statistical analysis of the study, emphasizing that the data are related to group averages, from where It would be important to examine the differences between participants to determine the moderating factors and characteristics of the respondents and non-respondents to the program. Thus, the intensity of the intervention differed between the groups. As a result, the greater improvements experienced by the ESDM group cannot be definitively attributed to the content of the intervention, but rather may be the result of the intervention plan.

\section{Conclusion}

The competency model is the result of a comprehensive intervention development behavior developed as a method to alter the developmental trajectory of autistic children. The aim 
was to help children become more socially oriented and develop other skills through social interactions with adults and their integration into a specific educational environment. The intervention is based on the principles of applied behavioral analysis, but aims to teach children skills in an institutional setting [9]. Several areas of development are both stimulate and teach skills taking into account the level of development of the child in each area. Children who participate in the ESDM are assigned a multidisciplinary treatment team [10-17] that assesses their progress, including individual learning objectives, are targeted during play sessions. The child's performance and skills are used to assess each child's progress until they are ready for intervention. A relatively new intervention, so that the whole search examination of its effects is somewhat limited. In cognitive improvement, language, coping skills and diagnosis controlled comparisons did not show gains. The perspectives of our research must be directed towards a promising intervention for young autistic children. Future research should include more methodologically rigorous studies as well as independent replication, reviews of the generalization of the skills taught and direct comparisons. The development of a frame of psychosocial skills that will be oriented to the good management and distribution of the knowledge of the educators in favor of the good integration and good communication of autistic subject.

\section{References}

1. Dawson G, Jones EJ, Merkle K, Lowy R, Faja S, et al. (2012) Early Behavioral Intervention is

2. Vismara L, McCormick C, Young G, Nadhan A, Monlux $K(2013)$ Preliminary findings of a telehealth approach to parent training in autism. Journal of Autism and Developmental Disorders 43: 2953-2969.

3. Eapen V, Crncec R, Walter A (2013) Clinical outcomes of an early intervention program

4. Vivanti G, Dissanayake C, Zierhut C, Rogers S, Victorial ASELCC Team (2013) Brief Report: Predictors of Outcomes in the Early Start Denver Model Delivered in a Group Setting.

5. Fulton E, Eapen V, Crncec R, Walter A, Rogers S (2014) Reducing maladaptive behaviors in preschool-aged children with autism spectrum disorder using the Early Start Denver. Front Pediatr 2:40.
6. Vismara L, Young G, Rogers S (2012) Telehealth for expanding the reach of early autism training to parents. Autism Research and Treatment 1-12.

7. Vismara L, Colombi C, Rogers S (2009 Can one hour per week of therapy lead to lasting changes in young children with autism?. Autism 13: 93-115.

8. Smith T, Iadarola $\mathrm{S}$ (2015) Achieving greater generalization of treatment effects in children with autism: Pivotal response training and self-management. Clinical Psychologist 46: 184-191.

9. Schreibman L (2005) The science and fiction of autism. Cambridge, MA: Harvard University Press.

10. Schreibman L, Dawson G, Stahmer A, Landa R, Rogers S, McGee, G., Kasari, C., Ingersoll, B., Kaiser, A., Bruinsma, Y., McNerney, E., Weatherby, A., \& Halladay, A. (2015).

11. Rogers SJ, Estes A, Lord C, Vismara L, Winter J, Fitzpatrick A, Dawson G (2012) Effects of a brief Early Start Denver Model (ESDM)-based parent intervention on toddlers at risk for autism spectrum disorders: a randomized controlled trial. Journal of the American Academy of Child and Adolescent Psychiatry 51: 10521065.

12. Volkmar R Paul A, Klin, D Cohen (Eds), Handbook of Autism and Developmental Disorders (3rd ed., Vol. 2, pp. 1055-1086.

13. Rogers SJ, Pennington BF (1991) A theoretical approach to the deficits in infantile autism. Development and Psychopathology 3: 137-162.

14. Rogers SJ, Vismara LA (2008) Evidence-Based Comprehensive Treatments for Early Autism. Journal of Clinical Child \& Adolescent Psychology 37: 8-38.

15. Schreibman L, Pierce KL (1993) Naturalistic Developmental Behavioral Interventions: Empirically Validated Treatments for Autism Spectrum Disorder. Journal of Autism and Developmental Disorders 45: 2411-2428.

16. Evidence Base Update for Autism Spectrum Disorder. Journalof Clinical Child \& Adolescent Psychology, 44(6), 897-922. UC Davis Extensions: Continuing and Professional Education. Retrieved April 10, 2016 from https://extension.ucdavis.edu/course/early-start-denvermodel-introductory-workshop

17. UC Davis MIND Institute ESDM Training Program. Retrieved April 9, 2016.

18. Dawson G, Rogers S, Munson J, Smith M, Winter JG, et al. (2010) Randomized, Controlled Trial of an Intervention for Toddlers with Autism: The Early Start Denver Model. Pediatrics 125: e17-123. 